\title{
Effect of a new health education model on continuous nursing in elderly patients with diabetes mellitus
}

Jing-Jing Chao, Ya-Zhuo Xue*

College of Nursing, Shandong Taishan Medical University, Tai'an, Shandong 271000, China

Received: 4 June 2017; Accepted: 27 June 2017; Published: 20 March 2018

Abstract: Objective: We aimed to explore the effect of a new health education model on continuous nursing care in elderly patients with diabetes mellitus who had undergone an operation for fracture.

Methods: Convenience sampling was used to select 59 elderly patients with diabetes mellitus and fracture. New health education methods were used, and patient parameters were evaluated before and after the intervention.

Results: Evaluation of medication, reasonable diet, regular exercise, blood glucose monitoring, and regular follow-up compliance were significantly improved in the experimental group compared to the control group $(P<0.05)$. There were also significant differences between groups in fasting blood glucose, 2-hour postprandial blood glucose, triglyceride, high-density lipoprotein, and low-density lipoprotein levels $(P<0.05)$; however, the differences between groups in terms of glycosylated hemoglobin and total cholesterol levels were not statistically significant $(P>0.05)$. Finally, the functional recovery and mental health of the experimental group were significantly better than those of the control group $(P<0.05)$.

Conclusions: The implementation of a menu of voluntary services in community-based continuous nursing provided standardized nursing care for elderly patients with fracture and diabetes mellitus and improved their quality of life.

Keywords: advanced age • continuous nursing • diabetes • fracture • health education • functional recovery

(c) Shanxi Medical Periodical Press.

\section{Introduction}

According to 2014 data of the National Bureau of Statistics, the population zaged 60 years in China has reached 177 million people, accounting for about $13.32 \%$ of the total population, indicating that the country has entered an aging stage. ${ }^{1}$ At the same time, diabetes is a common chronic disease in the elderly and its incidence is increasing annually. With age, calcium activity decreases, increasing the risk of fracture, especially of the femur neck, tibia, or ulna. Due to decreased physical function in patients with diabetes, bone fracture trauma can aggravate the condition and the risk of complications in this population. Compared to young patients, the recovery period following fracture is extended, seriously affecting the quality of life..$^{2,3}$
In addition, patients who experience disease education during their hospitalization remember only $60 \%$ of the knowledge upon discharge, which further decreases over time, resulting in reduced compliance with doctor's instructions. Therefore, targeted measures are necessary for these patients to improve their quality of life. ${ }^{4}$ At present, the change in the biomedical model has resulted in a change in the mode of health education from traditional teaching to patient-centered education. ${ }^{5}$ A menu-style volunteer service model of health education in Tai'an city provides volunteer service through an activities menu. The menu-style volunteer service refers to having certain professional volunteers collect information and present it in a volume, which is distributed to communities, villages, 
and towns, to allow residents to provide services such as volunteers to hotels/institutions that order these services. ${ }^{6,7}$ Through four steps, namely, preparing dishes, ordering, sending food, and tasting, as well as assessment of the condition of the object of study, the model aims to manage, evaluate, and improve the health education system; finally, a standardized voluntary service system is formed. . $^{8,9}$ This study, carried out from September 2016 to February 2017, assessed the effects of the implementation of a voluntary health education system in community elderly diabetes patients with fracture and provides an ethical review.

\section{Materials and methods}

\subsection{Study subjects}

This study used convenience sampling to select 59 cases of elderly patients with fracture and diabetes mellitus, who attended the Department of Orthopedic Surgery in a hospital of Tai'an City from September 2016 to February 2017. The patients ranged in age from 60 years to 86 years, with an average age of 77.5 years. Among them, there were six cases of fibular fracture, 11 cases of tibial fracture, nine cases of femoral fracture, 27 cases of radius fracture, and 12 cases of ulnar fracture. The history of diabetes ranged from 17 years to 1 year, and the patients' fasting blood glucose concentrations ranged from 8.3 to $16.4 \mathrm{mmol} / \mathrm{L}$. Among them, 37 cases had a history of diabetes and 12 cases were diagnosed with diabetes after hospitalization.

Finally, the patients were divided into experimental (33 cases) and control (26 cases) groups: foreign patients and patients for whom community nursing observation was not convenient were included in the control group, and patients residing in the city and patients for whom community nursing was convenient were included in the experimental group. There was no significant difference between the two groups in terms of age, sex, disease severity, and educational level $(P>0.05)$.

\subsection{Nursing interventions}

The nurses administered anti-inflammatory, oral hypoglycemic drugs, or insulin injections to the 59 patients after surgery, helped reduce local edema, provided diet guidance and early functional rehabilitation exercises, and established personal health registration forms.

\subsubsection{Control group: routine hospital health education}

On the day of discharge, health-care-related education was provided, to inform the patient of hospital procedures and matters requiring attention, by a responsible nurse according to the patient's condition and operational situation of the hospital.

(1) Blood glucose control: patients were informed of the correct use of hypoglycemic drugs according to the doctor's instructions, the need to regularly monitor blood sugar levels, and the adverse reactions to and countermeasures for hypoglycemic drugs.

(2) Diet guidance and incision care: because of the peculiarity of the diabetes diet, the importance of proper nutrition and the need to monitor blood glucose were emphasized. In addition, patients were informed and trained regarding the normal and abnormal conditions, treatments for wound healing, and how to prevent bedsores, deep vein thrombosis, pulmonary infection, and other complications. Finally, the patients were informed about the stitches and postoperative follow-up routine.

(3) A doctor-patient contact card was issued because patients with diabetes have poor peripheral circulation, which may lead to an extended period of time for functional recovery. It is easy to address patient problems by establishing an effective relationship between the nurse and the patient.

(4) Psychological guidance: psychological health education was provided to patients and their families in order to increase their confidence in overcoming the disease.

\subsubsection{Experimental group: routine hospital health education and menu-based health education}

In addition to the routine care provided to the control group, the observation group was also offered a voluntary health education menu, according to the Tai'an municipal civilization office guidelines, which was developed to meet the living needs of the community residents and improve their quality of life, as follows:

(1) The "meal preparation service" team consisted of members of four departments, including orthopedics specialists (two chief physicians and two residents), two diabetes specialists (two physicians), one psychologist, one public nutritionist, four nurse specialists (two from the Department of Orthopedics and two diabetes specialist nurses who earned the title after system specialist training), and two community health service center members of the medical staff. The titles and expertise of the 14 medical personnel were included in a booklet issued to the patients; the community health workers were responsible for answering patient questions and collecting basic information from patient records, including name, 
address, age, clinical diagnosis, surgery date, time to discharge, and contact information.

(2) Patients with disease-related service requirements could "Order" consultations in two ways:

(a) based on their needs and the menu information, patients could directly telephone a consultation doctor;

(b) patients could inform the community health care staff the name of the specialist and the knowledge they wanted to know, and experts from the community health care would schedule a health education lecture.

(3) "Send food": the implementation of the health education process mainly included two parts,

(a) volunteers who directly responded to patient phone calls;

(b) volunteers who provided community health education seminars. Before the lecture, community medical staff reported on patient questions and requests that suggested inadequate education. Five days in advance, the community nursing staff distributed a poster to the community publicity column and telephoned to inform the patients in the experimental group of the upcoming lecture. The volunteers used a variety of educational methods and topics, such as displaying the food model, enabling patients to correct ingestion methods on how much food there is in order to have an intuitive understanding, and providing a practical demonstration of insulin, among other lessons. At the end of each lecture, the patients' knowledge was assessed through a question-and-answer form and the patient was asked to share his/ her own nursing experience.

(4) "Food dishes": based on the information obtained from evaluations of the effect of health education in patients - provided by community health records and regular telephone follow-up, the menu consultation service for patients, and volunteer opinions and suggestions - additional guidance and education to help patient understanding were prepared for the future.

\subsection{Evaluation methods}

(1) Treatment compliance: diabetes treatment adherence was assessed based on the scale proposed by $\mathrm{Chen}^{10}$ in 2007, according to $\mathrm{Li}$ and Cai, ${ }^{11}$ who developed the diabetes compliance questionnaire; after repeated revision and expert consultation, the content validity was 0.83 , the test-retest reliability was 0.86 , and Cronbach's alpha was 0.86 . A total of five dimensions and 20 entries were used to evaluate patient compliance with medication, reasonable diet, regular exercise, blood glucose tests, and regular follow-up. Each item was scored as follows: regularly (one point), occasionally (two points), and never (three points). The total score ranges from 20 to 60 points, with a higher score indicating better compliance.

(2) Blood glucose and lipid levels: these measures included levels of fasting plasma glucose (FPG), 2-hour postprandial plasma sugar (2hPG), glycosylated hemoglobin $(\mathrm{HbA} 1 \mathrm{c})$, total cholesterol (TC), triglycerides (TGs), high-density lipoprotein-cholesterol (HDL-C), and low-density lipoprotein-cholesterol (LDL-C).

(3) Short musculoskeletal function assessment (SMFA) ${ }^{12}$ : the English version of the tool was developed by the University of Minnesota, while the Chinese version was developed by Wang ${ }^{13}$ in 2015. The questionnaire includes disability and annoyance indexes, with two subscales containing 34 and 12 entries, respectively. The scores of the two standardized subscales contain a total of 100 points, with a higher total score indicating a greater degree of dysfunction. All three indexes were measured at the beginning and 6 months after the intervention, respectively.

\subsection{Statistical methods}

The data were recorded in a Microsoft Excel spreadsheet and analyzed using IBM SPSS Statistics for Windows, version 20.0. Data were expressed as average \pm standard deviation $(M \pm S D)$, and the differences between the two groups were assessed using the twosample $t$-test $(\alpha=0.05)$.

\section{Results}

\subsection{Treatment compliance before and after intervention}

The patient compliance in terms of medication, reasonable diet, regular exercise, blood glucose monitoring, and regular follow-up improved significantly 
in the experimental group compared to those in the control group $(P<0.05)$. Analysis of blood glucose and lipid levels revealed significant changes in FPG, 2hPG, TG, HDL-C, and LDL-C levels in the experimental group $(P<0.05)$, while the changes in $\mathrm{HbA} 1 \mathrm{c}$ and TC did not change significantly between groups $(P>0.05)$ (Tables 1 and 2$)$.

\subsection{Comparison of blood glucose and blood lipid levels before and after the intervention}

Table 2 shows the changes in blood glucose and lipid levels before and after intervention in the control and experimental groups.

\subsection{Comparisons of dysfunction and annoyance indexes}

The dysfunction and annoyance indexes of the control and experimental groups before and after intervention are shown in Table 3.

\section{Discussion}

\subsection{Menu-based voluntary health education can improve patient compliance}

Research has shown that behavior change can be used to measure the success of diabetes education programs. ${ }^{14,15}$ In this study, the effects of the application of a menu-

\begin{tabular}{|c|c|c|c|c|c|c|c|c|}
\hline \multirow[b]{2}{*}{ Items } & \multicolumn{4}{|c|}{ Control group } & \multicolumn{4}{|c|}{ Experimental group } \\
\hline & $\begin{array}{c}\text { Before } \\
\text { intervention }\end{array}$ & $\begin{array}{c}\text { After } \\
\text { intervention }\end{array}$ & $t$ & $P$ & $\begin{array}{c}\text { Before } \\
\text { intervention }\end{array}$ & $\begin{array}{c}\text { After } \\
\text { intervention }\end{array}$ & $t$ & $P$ \\
\hline Medication compliance & $8.15 \pm 1.38$ & $8.08 \pm 1.41$ & 0.40 & 0.07 & $8.06 \pm 1.17$ & $8.61 \pm 1.62$ & -2.25 & $0.032^{\#}$ \\
\hline Reasonable diet & $7.69 \pm 1.19$ & $7.88 \pm 1.18$ & -1.55 & 0.13 & $7.55 \pm 1.06$ & $9.00 \pm 1.80$ & -4.11 & $0.000^{*}$ \\
\hline Regular exercise & $7.85 \pm 0.97$ & $8.15 \pm 1.41$ & -1.03 & 0.31 & $7.76 \pm 1.12$ & $8.70 \pm 1.68$ & -2.70 & $0.011^{\#}$ \\
\hline Blood glucose monitoring & $7.50 \pm 1.27$ & $7.77 \pm 1.45$ & -1.77 & 0.09 & $7.21 \pm 1.19$ & $8.61 \pm 1.71$ & -4.21 & $0.000^{*}$ \\
\hline Periodic inspection & $7.11 \pm 1.14$ & $7.34 \pm 1.05$ & -1.81 & 0.08 & $7.09 \pm 1.21$ & $8.21 \pm 1.50$ & -3.77 & $0.01 *$ \\
\hline
\end{tabular}

Table 1. Patient compliance before and after the intervention $(M \pm S D, n=59)$

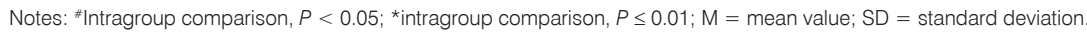

\begin{tabular}{c|cccccccc}
\hline \multirow{2}{*}{ Items } & \multicolumn{3}{|c}{ Control group } & \multicolumn{3}{c}{ Experience group } \\
\cline { 2 - 8 } & Before intervention & After intervention & $t$ & $P$ & Before intervention & After intervention & $t$ \\
\hline \hline FPG & $8.88 \pm 1.56$ & $8.38 \pm 1.12$ & 1.443 & 0.161 & $8.96 \pm 0.99$ & $8.29 \pm 0.61$ & 3.747 & $0.001^{*}$ \\
2hPG & $13.97 \pm 2.10$ & $13.39 \pm 2.48$ & 1.81 & 0.083 & $13.51 \pm 2.08$ & $12.95 \pm 2.37$ & 2.18 & $0.037^{\#}$ \\
HbA1C & $8.315 \pm 0.59$ & $8.18 \pm 0.62$ & 2.023 & 0.054 & $8.45 \pm 0.60$ & $8.30 \pm 0.59$ & 1.964 & 0.058 \\
TC & $4.52 \pm 0.33$ & $4.44 \pm 0.29$ & 1.899 & 0.069 & $4.59 \pm 0.36$ & $4.52 \pm 0.33$ & 2.01 & 0.053 \\
TG & $1.63 \pm 0.27$ & $1.57 \pm 0.17$ & 1.509 & 0.144 & $1.68 \pm 0.30$ & $1.57 \pm 0.15$ & 2.212 & $0.034^{\#}$ \\
HDL-C & $0.83 \pm 0.29$ & $0.91 \pm 0.38$ & 1.443 & 0.161 & $0.81 \pm 0.26$ & $0.92 \pm 0.34$ & 2.517 & $0.017^{\#}$ \\
LDL-C & $3.81 \pm 0.38$ & $3.69 \pm 0.35$ & 1.995 & 0.057 & $3.72 \pm 0.41$ & $3.59 \pm 0.39$ & 2.62 & $0.013^{\#}$ \\
\hline
\end{tabular}

Table 2. Blood glucose and blood lipid changes $(\mathrm{M} \pm \mathrm{SD}, n=59)$

Notes: "Intragroup comparison, $P<0.05$; *intragroup comparison, $P<=0.01 ; 2 \mathrm{hPG}=2$-hour postprandial plasma sugar; $\mathrm{FPG}=$ fasting plasma glucose; $\mathrm{HbA} 1 \mathrm{C}=$ glycosylated hemoglobin; HDL-C = high-density lipoprotein-cholesterol; LHDL-C = low-density lipoprotein-cholesterol; $\mathrm{M}=$ mean value; $\mathrm{SD}=\mathrm{standard}$ deviation; TC = total cholesterol; TG = triglyceride.

\begin{tabular}{|c|c|c|c|c|c|c|c|c|}
\hline \multirow[b]{2}{*}{ Items } & \multicolumn{4}{|c|}{ Control group } & \multicolumn{4}{|c|}{ Experimental group } \\
\hline & $\begin{array}{c}\text { Before } \\
\text { intervention }\end{array}$ & After intervention & $t$ & $P$ & $\begin{array}{c}\text { Before } \\
\text { intervention }\end{array}$ & After intervention & $t$ & $P$ \\
\hline $\begin{array}{l}\text { Disability } \\
\text { index }\end{array}$ & $67.42 \pm 7.12$ & $65.5 \pm 5.98$ & 1.995 & 0.057 & $69.36 \pm 6.82$ & $66.69 \pm 7.12$ & 2.153 & $0.039^{\#}$ \\
\hline $\begin{array}{c}\text { Annoyance } \\
\text { index }\end{array}$ & $70.11 \pm 6.92$ & $67.81 \pm 7.19$ & 1.806 & 0.083 & $70.58 \pm 7.65$ & $66.09 \pm 7.54$ & 2.799 & $0.009^{*}$ \\
\hline
\end{tabular}

Table 3. Comparisons of dysfunction and annoyance indexes $(M \pm S D, n=59)$

Notes: "Intragroup comparison, $P<0.05$; *Intragroup comparison, $P<=0.01 ; \mathrm{M}=$ mean value; $\mathrm{SD}=$ standard deviation. 
based volunteer health education in community patients with diabetes on compliance are shown in Table 1. Patient compliance was significantly higher after the intervention $(P<0.05)$. The reasons for this finding may be the following:

(1) the "delivery" process, which used a variety of ways to provide volunteer education that allowed patients with diabetes in the community to participate actively in the nursing process of the disease; through the study of related knowledge, patients were better able to understand the importance of diet, exercise, medication compliance, and blood glucose monitoring;

(2) the "send food" process, in which diabetes specialist nurses regularly telephoned for follow-up assessments to better understand the patients' condition and supervise the patients' adoption of healthy behaviors.

\subsection{Menu-based voluntary health education can improve blood sugar and blood lipid levels}

Table 2 shows that the voluntary service menu health education intervention reduced the FPG, 2hPG, TG, and LDL-C levels and increased HDL-C level $(P<0.05)$, which may be related to health education that improved compliance, modified poor habits, and encouraged a reasonable diet and regular exercise. The $\mathrm{HbA} 1 \mathrm{c}$ and TC levels tended to decrease, but the difference was not significant $(P>0.05)$, which might be related to the short intervention period.

\subsection{Intervention can promote the recovery of patients' function and improve their mental health}

Table 3 shows that the menu service for nursing health education promoted the functional recovery of patients $(P<0.05)$, facilitated fracture recovery to progress to isotonic and isometric exercise, and increased patient confidence to overcome the disease $(P<0.05)$ in the experimental group, compared to the control group, in order to improve patient mental health.

\section{Conclusions}

The implementation of a menu service for patient health education following hospital discharge can provide more comprehensive and more specific health guidance, which may promote functional recovery, improve mental health, and improve the quality of life of patients, as well as reduce the economic burden on the family and save valuable medical resources, which are conducive to the development of social security system and health improvement. ${ }^{16}$ Therefore, comprehensive hospitals and community medical institutions should work together in order to establish the continuity of the medical system. However, the establishment of this method to improve the long-term care of the elderly patients will require government participation, increased investment, improved community facilities, and increased medical staff ratios in order to offer education for elderly patients for disease treatment and prevention. The present study had several limitations, including the limited implementation time and patient conditions, as well as the fact that the patient menu-based health education service is a volunteerbased activity; whether the findings of the present study can be applied to other community patients with chronic diseases requires further research.

\section{Conflicts of interest}

All contributing authors declare no conflicts of interest.

\section{References}

1. American Diabetes Association. Promoting health and reducing disparities in populations. Diabetes Care. 2017;40(suppl 1):S6-S10.

2. Ensrud KE, Ewing SK, Taylor BC, et al. Comparison of 2 frailty indexes for prediction of falls, disability, fractures, and death in older women. Arch Intern Med. 2008;168:382-389.

3. Ensrud KE, Ewing SK, Cawthon PM, et al. A comparison of frailty indexes for the prediction of falls, disability, fractures, and mortality in older men. J Am Geriatr Soc. 2009;57:492-498.

4. Adikusuma W, Qiyaam N. The effect of education through Short Message Service (SMS) messages on diabetic patients adherence. Sci Pharm. 2017;85:pii: E23.

5. Qiu YH, Wang J, Zhou FY. Development trend and strategy of health medicine. Chin Med Sci. 2015;2:214-216. (in Chinese).

6. Barasheh N, Shakerinejad G, Nouhjah S, Haghighizadeh $\mathrm{MH}$. The effect of educational program based on the precede-proceed model on improving self-care behaviors in a semi-urban population with type 2 diabetes referred to health centers of Bavi, Iran. Diabetes Metab Syndr. 2017;11(suppl 2):S759-S765.

7. Sousa FA, Goulart MJ, Braga AM, et al. Setting health priorities in a community: a case example. Rev Saude Publica. 2017;51:11. 
8. Haas L, Maryniuk M, Beck J, et al. National standards for diabetes self-management education and support. Diabetes Educ. 2012;38:619-629.

9. Gee PM, Greenwood DA, Paterniti DA, Ward D, Miller LM. The eHealth Enhanced Chronic Care Model: a theory derivation approach. J Med Internet Res. 2015;17:e86.

10. Chen YM. Investigation and Research on the Compliance of Diabetic Patients in Readmission Hospital and its Influencing Factors. Changsha: School of Nursing, Central South University; 2007:4-5. (in Chinese).

11. Li XQ, Cai HW. Investigation of compliance behavior in diabetes mellitus. Chin J Nurs. 2004;39:500502. (in Chinese).

12. van Delft-Schreurs CCHM, van Son MAC, de Jongh $M A C$, et al. The relationship between physical and psychological complaints and quality of life in severely injured patients. Injury. 2017;48:1978-1984.
13. Wang Y. Evaluation of the Chinese Version of Musculoskeletal Functional Assessment Based on Classical Measurement Theory and Item Response Theory. Guangzhou: Southern Medical University; 2015. (in Chinese).

14. Peeples M, Mulcahy K, Tomky D, Weaver T; National Diabetes Education Outcomes System (NDEOS). The conceptual framework of the National Diabetes Education Outcomes System (NDEOS). Diabetes Educ. 2001;27:547-562.

15. Gildea CM, Lantaff WM, Olenik NL. Identifying barriers to glycemic control in patients with type 2 diabetes after completion of an accredited education program. J Am Pharm Assoc. (2003). 2017;57:S211-S216.

16. Yan F. Study on the Improvement of Primary Medical and Health Service System in the Background of Population Aging - Taking Xinbei District of Changzhou as an Example. Suzhou: Soochow University; 2014. (in Chinese).

How to cite this article: Chao JJ, Xue YZ. Effect of a new health education model on continuous nursing in elderly patients with diabetes mellitus. Frontiers Nurs. 2018; 1: 69-74.https://doi.org/10.1515/fon-2018-0010 